\title{
Decreased expression of $L R A 4$, a key gene involved in rhamnose metabolism, caused up-regulated expression of the genes in this pathway and autophagy in Pichia pastoris
}

\author{
Jian Jiao ${ }^{\dagger}$, Shuai Wang ${ }^{\dagger}$, Hui Tian, Xinxin Xu, Yuhong Zhang, Bo Liu ${ }^{*}$ and Wei Zhang
}

\begin{abstract}
In a previous study, we developed Pichia pastoris GS115m, an engineered strain with decreased expression of one key gene, $L R A 4$, in rhamnose metabolism. P. pastoris GS115m/LacB was subsequently constructed via introducing a $\beta$-galactosidase gene, $L A C B$, under the control of rhamnose-inducible $P_{L R A 3}$ into P. pastoris GS115m. P. pastoris GS115m/LacB greatly improved recombinant protein production relative to the parental strain (P. pastoris GS115/LaCB). In the present study, transcriptomes of P. pastoris GS115m/LacB and P. pastoris GS115/LacB grown in YPR medium were analyzed. P. pastoris GS115m/LaCB was found to suffer from the mild carbon source starvation. To attenuate the starvation stress, $P$. pastoris GS115m/LacB attempted to enhance rhamnose metabolism by elevating the transcription levels of rhamnose-utilization genes $\angle R A T-3$ and RhaR. The transcription level of $\angle a C B$ under the control of $\mathrm{P}_{L R A 3}$ thereby increased, resulting in the improved production of recombinant protein in P. pastoris GS115m/LacB. It was also revealed that $P$. pastoris GS1 $15 \mathrm{~m} / \mathrm{LaCB}$ cells coped with carbon starvation mostly via autophagy.
\end{abstract}

Keywords: Pichia pastoris, Rhamnose metabolism, Transcriptome, Autophagy

\section{Introduction}

Because of its toxic and flammable properties, the use of methanol is dangerous during recombinant protein production. Hence, safe compound-inducible promoters are needed as alternatives to the methanol-inducible promoter $\mathrm{P}_{A O X 1}$ in Pichia pastoris expression systems (Vogl and Glieder 2013). Several strong and inducible promoters, such as $\mathrm{P}_{\mathrm{PHO} 9}$ or $\mathrm{P}_{T H I 11}$, have been discovered to date (Ahn et al. 2009; Stadlmayr et al. 2010). Extensive efforts have been made in our laboratory to identify some other alternatives to $\mathrm{P}_{A O X 1}$, and we have discovered several rhamnose utilization related genes (four enzyme-coding genes, $L R A 1-4$, and one regulator-coding gene, RhaR).

\footnotetext{
*Correspondence: liubo01@caas.cn; zw_bio@caas.cn

${ }^{\dagger}$ Jian Jiao and Shuai Wang contributed equally to this work Biotechnology Research Institute, Chinese Academy of Agricultural Sciences, No.12., Zhongguancun South St., Haidian District, Beijing 100081, China
}

Simultaneously, the promoters of $L R A 3$ and $L R A 4, \mathrm{P}_{L R A 3}$ and $\mathrm{P}_{L R A 4}$, were identified as two strong rhamnoseinducible promoters (Jiao et al. 2019; Liu et al. 2016). Subsequently, a Pichia expression system based on $\mathrm{P}_{L R A 3}$, designated as the $\mathrm{P}_{L R A 3}$ system, was developed using rhamnose as the inducer. However, the $\mathrm{P}_{L R A 3}$ system did not produce recombinant proteins as efficiently as the $\mathrm{P}_{A O X 1}$ system. To enhance recombinant protein production in the $\mathrm{P}_{L R A 3}$ system, the engineered strain P. pastoris GS115m with decreased rhamnose metabolism flux was constructed via replacing the strong rhamnose-inducible promoter $\mathrm{P}_{L R A 4}$ by another weak rhamnose-inducible promoter $\mathrm{P}_{L R A 2}$. P. pastoris $\mathrm{GS} 115 \mathrm{~m}$ presented several different profiles compared to the parental strain $P$. pastoris GS115 as follows: (i) lower rhamnose utilization rate due to decreased expression of $L R A 4$, (ii) reduced cell biomass and growth rate, and (iii) improved recombinant protein production (Yan et al. 2018). Additionally, the 
engineered strain exhibited flocculation and rapid sedimentation at high cell densities $\left(\mathrm{OD}_{600}>6\right)$ when it was grown in rhamnose-containing media, particularly YPR medium (Yan et al. 2018).

There are several possible explanations for the different physiological profiles of the engineered strain from the parental strain. Theoretically, down-regulating the expression of $L R A 4$, which encodes a rate-limited enzyme involved in rhamnose metabolism, should reduce the utilization efficiency of rhamnose in the engineered strain cultured in rhamnose-containing media (e.g., YPR). In turn, this may result in mild carbon starvation stress due to insufficient rhamnose utilization. Subsequently, numerous physiological profiles including cell viability, autophagy and cell apoptosis, which were reported to be subject to carbon starvation stress (Oda et al. 2015; Weidberg et al. 2011; Wang et al. 2018b), would be altered in the engineered strain to adapt to the mild carbon starvation stress. To verify these speculations and shed light on the related responses, in this study we investigated the differences in transcriptomes and several physiological profiles between the engineered strain P. pastoris $\mathrm{GS} 115 \mathrm{~m} / \mathrm{LacB}$ and the parental strain P. pastoris GS115/LacB during growth on rhamnose. According to the evidence, we elucidated the molecular mechanism for the improved production of recombinant protein in $P$. pastoris GS115m/LacB. Simultaneously, it was disclosed that $P$. pastoris $\mathrm{GS} 115 \mathrm{~m} / \mathrm{LacB}$ coped with the mild carbon source starvation mostly via elevating autophagy level. The results would help to understand the survival mechanism responsible for starvation stress including but not limited to carbon source starvation and simultaneously provide a novel strategy for engineering strain to improve produce of target products.

\section{Materials and methods}

\section{Strains and medium}

Pichia pastoris GS115m was developed from P. pastoris GS115 by replacing the strong rhamnose-inducible promoter $\mathrm{P}_{L R A 4}$ with the weak rhamnose-inducible promoter $\mathrm{P}_{L R A 2}$. In the $P$. pastoris $\mathrm{GS} 115 / L a c B$ and $P$. pastoris GS115m/LacB strains, the $\beta$-galactosidase coding gene $L a c B$ is under the control of the rhamnose-inducible promoter $\mathrm{P}_{L R A 3}$. All strains were described in detail in a previous study (Yan et al. 2018).

The YPD medium contained $1 \%$ yeast extract, $2 \%$ peptone, and $2 \%$ dextrose. The YPR medium contained $1 \%$ yeast extract, $2 \%$ peptone, and $2 \%$ rhamnose. The MR medium contained $1.34 \%$ yeast nitrogen base, $4 \times 10^{-5} \%$ biotin, and $2 \%$ rhamnose. To prepare solid medium, agar was supplemented into the above media to a final concentration of $2 \%$.

\section{Production determination of recombinant protein} Cultivation of $P$. pastoris $\mathrm{GS} 115 / \mathrm{LacB}$ or $P$. pastoris $\mathrm{GS} 115 \mathrm{~m} / \mathrm{LacB}$ in YPR medium and analysis of $\beta$-galactosidase activities in the culture supernatants were carried out as previously described (Yan et al. 2018). The wet cell weight (WCW) per milliliter of culture was simultaneously determined at intervals. The $\beta$-galactosidase productivity per $\mathrm{mg}$ of $\mathrm{WCW}$ was assayed based on $\beta$-galactosidase activities in the culture supernatants, specific activity of $\beta$-galactosidase (575 U/ $\mathrm{mg})$, and WCW.

\section{Total RNA preparation for RNA-seq and real-time polymerase chain reaction (PCR)}

Each strain (P. pastoris GS115/LacB and P. pastoris $\mathrm{GS} 115 \mathrm{~m} / \mathrm{LacB}$ ) was inoculated into YPR medium and grown until an $\mathrm{OD}_{600}$ of $\sim 2$ or $\sim 6$ was reached. The cells were collected using centrifugation $(12,000 \mathrm{~g})$ at $4{ }^{\circ} \mathrm{C}$ for 4 min and stored at $-80^{\circ} \mathrm{C}$ before total RNA extraction. Total RNA extraction and trace DNA removal were performed according to previously described methods (Liu et al. 2016). RNA concentration was determined with a Qubit $^{\circledR} 2.0$ fluorometer (Life Technologies, CA, USA), and the quality was checked using an Agilent RNA 6000 Nano kit combined with an Agilent 2100 Bioanalyzer (Santa Clara, CA, USA).

\section{RNA sequencing and RNA-seq data analysis}

An RNA-seq library was constructed using the NEBNext ${ }^{\circledR}$ Ultra $^{\mathrm{TM}}$ RNA Library Prep kit for Illumina ${ }^{\circledR}$ (New England Biolabs, Ipswich, MA, USA) according to the manufacturer's instructions. RNA library quality confirmation and RNA sequencing were carried out according to the process described by Wang et al. (2018a).

Clean reads were obtained from the raw data by removing low-quality bases $(<\mathrm{Q} 20)$ using Trim_galore (Bolger et al. 2014). The mapping of clean reads onto the reference genome of $P$. pastoris GS115 was performed using TopHat (v2.0.12) (Trapnell et al. 2009). The strand-specific and unique mapped reads were analyzed using HTSeq (v0.6.1) (Anders et al. 2015). Fragments per kilobase of transcript sequence per millions base pairs sequenced (FPKM) was used to assay the abundance of each gene (Trapnell et al. 2010). Differentially expressed genes (DEGs) were identified using DEseq 2 with an FDR-adjusted $p$ value of $<0.05$ and the fold-change $\geq 2$.

\section{Real-time PCR}

Real-time PCR assays were performed using previously described methods (Yan et al. 2018). The primers used for real-time PCR are listed in Additional file 1: Table S1. The relative expression level of each test gene in $P$. pastoris 
GS115/LacB was assigned as 1, and the glyceraldehyde3-phosphate dehydrogenase (GAPDH) gene was used as a reference gene. Each gene was analyzed in triplicate, and the data are represented as the means $\pm(\mathrm{SD})$ standard deviations.

\section{Cell viability assay}

Cells of P. pastoris GS115/LacB (WT) and P. pastoris GS115m/LacB (MT) were grown in liquid YPR medium to an $\mathrm{OD}_{600}$ of $\sim 6$. Cultures were diluted to an $\mathrm{OD}_{600}$ of $\sim 1$ and then washed. After serial dilution (1: 10), $5 \mu \mathrm{L}$ of samples was spotted on YPR media and $10 \mu \mathrm{L}$ of samples were spotted on MR media, respectively. The colonies were observed after incubation at $28^{\circ} \mathrm{C}$ for $72 \mathrm{~h}$.

\section{Cell apoptosis analysis}

The Annexin V-FITC/PI Cell Apoptosis Detection Kit (TransGen, Beijing, China) was used for Annexin V staining. Cultures of P. pastoris GS115/LacB and P. pastoris $\mathrm{GS} 115 \mathrm{~m} / \mathrm{LacB}$ were diluted to an $\mathrm{OD}_{600}$ of $\sim 0.5$ after grown in YPR medium to an $\mathrm{OD}_{600}$ of $\sim 6$, and the cells were washed twice with PBS. Subsequently, the cells were incubated in $100 \mu \mathrm{L}$ of sorbitol buffer $(0.1 \mathrm{M}$ sodium phosphate buffer, $1.2 \mathrm{M}$ sorbitol, $\mathrm{pH}$ 7.4) containing 50 U Lyticase (TianGen, Beijing, China) at $28^{\circ} \mathrm{C}$ for $30 \mathrm{~min}$. After centrifugation $\left(800 g, 4{ }^{\circ} \mathrm{C}, 5 \mathrm{~min}\right)$, cells were washed with $500 \mu \mathrm{L}$ of $1.2 \mathrm{M}$ sorbitol buffer, and then resuspended in $100 \mu \mathrm{L}$ of Annexin V binding buffer containing $5 \mu \mathrm{L}$ of Annexin V-FITC and $5 \mu \mathrm{L}$ of PI, followed by incubation at room temperature for $15 \mathrm{~min}$. Later, the cells were washed once in $200 \mu \mathrm{L}$ of Annexin V binding buffer and resuspended in $100 \mu \mathrm{L}$ of Annexin V binding buffer. Cells were immediately visualized by laser scanning confocal microscope at a $488 \mathrm{~nm}$ excitation wavelength, and fluorescence intensities from $\sim 20,000$ cells were determined by flow cytometry (BD LSRFortessa).

\section{Staining of autophagosomes}

Cell Meter ${ }^{\mathrm{TM}}$ Autophagy Assay Kit "Blue Fluorescence* (AAT Bioquest, Sunnyvale, California, USA) was used for autophagosomes staining. P. pastoris GS115/LacB or $P$. pastoris $\mathrm{GS} 115 \mathrm{~m} / \mathrm{LacB}$ was diluted to an $\mathrm{OD}_{600}$ of $\sim 0.5$ when the cells were grown in YPR medium to an $\mathrm{OD}_{600}$ of $\sim 6$. The cells were collected by centrifuge and washed twice with PBS, and then were incubated in $500 \mu \mathrm{L}$ of stain buffer with $1 \mu \mathrm{L} 500 \times$ autophagy blue ${ }^{\mathrm{TM}}$ at $28{ }^{\circ} \mathrm{C}$ for $60 \mathrm{~min}$. After incubated the cells were washed twice with $500 \mu \mathrm{L}$ of washing buffer at $800 \mathrm{~g}$ for $5 \mathrm{~min}$ at $4{ }^{\circ} \mathrm{C}$, and then resuspended in $100 \mu \mathrm{L}$ of washing buffer. Cells were immediately visualized by laser scanning confocal microscope.

\section{Detection of reactive oxygen species (ROS)}

Intracellular levels of ROS were measured with ROS Assay Kit (BioDee, Beijing, China). P. pastoris GS115/LacB and P. pastoris GS115m/LacB were grown in YPR medium to an $\mathrm{OD}_{600}$ of $\sim 6$. The cells were diluted to $\mathrm{OD}_{600} \sim 0.5$. Collected and washed the cells twice with PBS incubated $30 \mathrm{~min}$ at $30^{\circ} \mathrm{C}$ with $30 \mu \mathrm{M}$ of dihydrorhodamine 123 (DHR123). After centrifugation (800g, $5 \mathrm{~min}, 4^{\circ} \mathrm{C}$ ), the cells were washed with $1 \mathrm{~mL}$ of PBS and resuspended in $100 \mathrm{~mL}$ of PBS, and then were observed using laser scanning confocal microscope at a $488 \mathrm{~nm}$ excitation wavelength and an emission wavelength shifting from green $(\sim 525 \mathrm{~nm})$, and fluorescence intensities from $\sim 20,000$ cells were monitored by flow cytometry.

\section{Results}

Recombinant protein production in P. pastoris GS115/LaCB and P. pastoris GS115m/LacB

In a previous study, growth rate and maximal biomass were lower for P. pastoris GS115m/LacB grown in YPR than for P. pastoris GS115/LacB. However, the amount of recombinant protein, $\beta$-galactosidase encoded by $L a c B$, in the supernatants of $P$. pastoris GS115m/LacB cultures was higher than that of $P$. pastoris GS115/LacB (Yan et al. 2018). This indicated that P. pastoris GS115m/LacB produced the target protein more efficiently. To verify this result, the production of recombinant protein in the two strains grown in YPR medium was investigated based on protein production $v s$ wet cell weight (WCW). The results revealed that Pichia cells efficiently produced target proteins from 12 to $36 \mathrm{~h}$, and the recombinant protein production at $72 \mathrm{~h}$ was more than twofold higher in P. pastoris GS115m/LacB cell than in P. pastoris GS115/LacB (Fig. 1). The underlying mechanisms for the improved production of recombinant protein, which

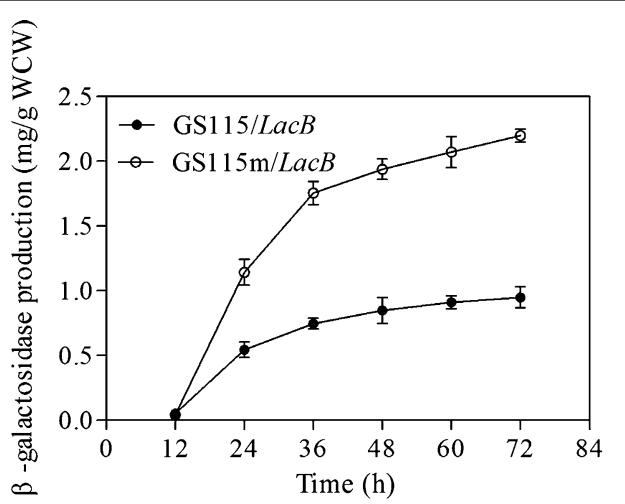

Fig. $1 \beta$-Galactosidase production of the test strains cultured in YPR medium. WCW wet cell weight 
could provide some important information for strain engineering, were further investigated.

\section{Global transcriptional profiles of $P$. pastoris GS115/LaCB and P. pastoris GS115m/LacB}

Pichia pastoris GS115/LacB and P. pastoris GS115m/LacB were cultured in YPR medium until an $\mathrm{OD}_{600}$ of $\sim 2$ or $\sim 6$, respectively. The cells from two biological replicates of $P$. pastoris GS115/LacB and P. pastoris $\mathrm{GS} 115 \mathrm{~m} / \mathrm{LacB}$ were collected, and total RNA from these cells was prepared for transcriptome analysis using RNA-seq. The RNA-seq data were deposited in the CNGBdb (https://db.cngb.org) with Accession Number CNP0000622 and CNP0000710. The overall expression levels in the two biological replicates of each group were highly similar to each other ( $R^{2}$ beyond 0.98 ) (Additional file 2: Fig. S1). At an $\mathrm{OD}_{600}$ of $\sim 2$, a total of 749 DEGs including 281 down-regulated and 468 up-regulated genes were identified among 5,041 genes, including an exogenous gene, $L a c B$. The large number of DEGs indicated that the decrease in rhamnose metabolic flux exerted wide-ranging effects on the transcriptomes of Pichia cells and thereby led to various changes in physiological profiles.

To further examine gene expression profiles, real-time PCR was performed to investigate the relative expression of four up-regulated DEGs (PAS_chr4_0550, PAS chr1-1_0356, PAS_chr3_0798, and PAS_chr4_0146), four down-regulated DEGs (PAS_chr3_0095, PAS_chr3_0403, PAS_chr4_0799, and PAS_chr3_0257), one non-DEG (PAS_chr3_0229), and two genes of interest (LRA4 and $L a c B)$. The trends in expression of the target genes were consistent between real-time PCR and RNA-seq despite the presence of minor differences in the expression levels of certain genes between the two methods (Fig. 2).

\section{Improved expression of $L R A 3$ in P. pastoris GS115m/LacB}

Highly transcribed genes usually play crucial roles in organism survival, and their expression changes under different conditions. According to the FPKM value of each gene, the 25 most highly expressed genes in P. pastoris GS115/LacB and P. pastoris GS115m/LacB were identified at an $\mathrm{OD}_{600}$ of $\sim 2$ (Additional file 3: Table S2) and an $\mathrm{OD}_{600}$ of $\sim 6$ (Additional file 4: Table S3), respectively. Theoretically, these genes are expected to play important roles in the survival of Pichia cells using rhamnose as the sole carbon source.

Notably, LRA3 was also one of the 25 most highly expressed genes in both strains grown to $\mathrm{OD}_{600} \sim 2$ and $\sim 6$, which suggested that $L R A 3$ expression was intensively induced in the presence of rhamnose. At $\mathrm{OD}_{600}$ of $\sim 2$ and $\sim 6$, the $L R A 3$ transcription level was ranked 21st and 14th in P. pastoris GS115/LacB, and while it was

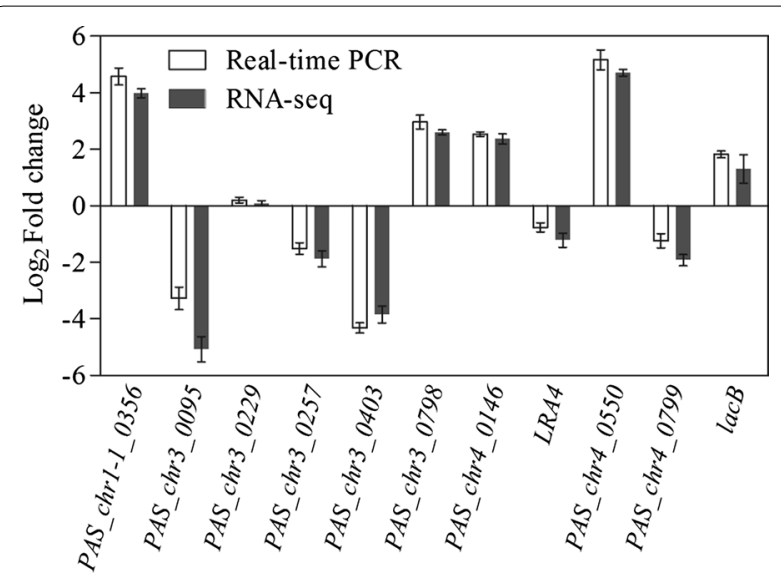

Fig. 2 Correlation of RNAseq and real-time PCR.PAS_chr1-1_0356, hypothetical protein; PAS_chr3_0095, phenylpyruvate decarboxylase; PAS_chr3_0229, hypothetical protein; PAS_chr3_0257, adenylate kinase; PAS_chr3_0403, Acetyl-coA synthetase isoform; PAS_chr3_0798, hypothetical protein; PAS_chr4_0146, hypothetical protein; LRA4, L-2-keto-3-deoxyrhamnonate aldolase; PAS_chr4_0550, hypothetical protein; PAS_chr4_0799, mitochondrial 54S ribosomal protein YmL7/ $Y \mathrm{~mL} 5 ; \mathrm{LaCB}, \beta$-galactosidase. The corresponding gene in $P$. pastoris GS115/LaCB was used as the control, and the relative expression value of each gene was designated as 1

ranked 2nd and 6th in P. pastoris GS115m/LacB, respectively. These results showed that $L R A 3$ expression level was significantly elevated in $P$. pastoris $\mathrm{GS} 115 \mathrm{~m} / \mathrm{LacB}$ with rhamnose induction. In addition, it was surprising that the $L R A 3$ transcription level was even higher than that of GAPDH in P. pastoris GS115m/LacB.

\section{Transcription profiles of the genes related to rhamnose metabolism during incubation}

Rhamnose was the main carbon source for cell survival when Pichia cells were grown in YPR medium, and the rhamnose utilization rate was therefore a key factor affecting energy production, biomass biogenesis, and physiological profiles in Pichia cells. Down-regulating the expression of key rate-determining step enzymes such as LRA4 would decrease rhamnose utilization efficiency, resulting in insufficient supply of energy and carbon matrices for primary and secondary metabolism, growth, and propagation. To adapt to these conditions, the strain should up-regulate the expression of rhamnose-utilization genes to accelerate rhamnose metabolism. This expectation was borne out by transcriptome analysis.

The genes involved in rhamnose metabolism included five genes such as four enzyme-coding genes (LRA1-4) and a regulator-coding gene $(R h a R)$. As expected, the expression levels of all the genes except for $L R A 4$ in P. pastoris $\mathrm{GS} 115 \mathrm{~m} / \mathrm{LacB}$ were differentially up-regulated more than twofold compared with $P$. pastoris 


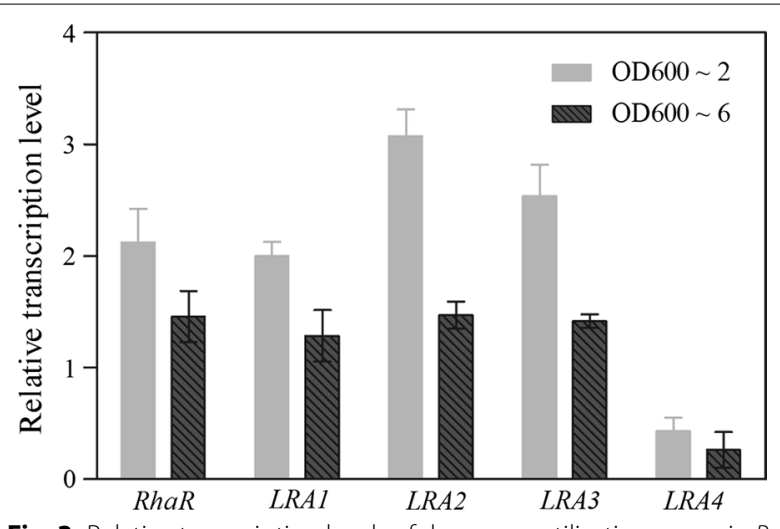

Fig. 3 Relative transcription levels of rhamnose utilization genes in $P$. pastoris GS115m/LacB grown in YPR medium. Four genes rhamnose utilization of $\angle R A 1, \angle R A 2, R h a R, \angle R A 3$, and $\angle R A 4$ was detected in $P$. pastoris GS115m/LacB grown in YPR medium to $\mathrm{OD}_{600} \sim 2$ and $\mathrm{OD}_{600} \sim 6$, respectively. The corresponding gene in P. pastoris GS115/LaCB was used as the control, and the relative expression value of each gene was designated as 1

GS115/LacB at an $\mathrm{OD}_{600}$ of $\sim 2$ (Fig. 3). Simultaneously, $L R A 4$ maintained its low expression as it was under the control of the weak promoter $\mathrm{P}_{L R A 2}$ (Fig. 3). These results indicated that the low production rate of the key rhamnose metabolism-related enzyme LRA4 led to inadequate production of energy and carbon matrices required for normal growth, and P. pastoris GS115m/LacB therefore enhanced the expression of rhamnose metabolismrelated genes to increase rhamnose utilization to provide more energy and biomass for cell growth.

To further investigate the expression profiles of these genes during incubation, the genes were examined when the strains were grown to a high cell density $\left(\mathrm{OD}_{600} \sim 6\right)$. Relative expression of all genes, except for $L R A 4$, decreased compared with that at an $\mathrm{OD}_{600}$ of $\sim 2$ (Fig. 3). When grown to $\mathrm{OD}_{600} \sim 12$, the expression levels of these genes in $P$. pastoris GS115m/LacB were almost equal to those of $P$. pastoris GS115/LacB (with the exception of $L R A 4$ ), which was reported previously (Yan et al. 2018). This could be explained that the decreasing concentration of residual rhamnose in the medium made a declining induction to the related gene expression. Overall, the relative expression of genes other than $L R A 4$ was dynamic; it was closely associated with the concentration of residual rhamnose in the medium and decreased with the consumption of rhamnose.

\section{$L a c B$ expression profiles during incubation}

Recombinant protein production, an important index for an expression system, was closely and positively dependent on the transcription activity of its promoter. In $P$. pastoris $\mathrm{GS} 115 \mathrm{~m} / \mathrm{LacB}, \mathrm{LacB}$ expression was controlled under $\mathrm{P}_{L R A 3}$, and thereby the production of the recombinant protein, $\beta$-galactosidase encoded by $L a c B$, was largely subject to the transcriptional activity of $\mathrm{P}_{L R A 3}$. As mentioned above, $L R A 3$ was one of the most highly transcribed genes, and $L R A 3$ expression was greatly enhanced in $P$. pastoris GS115m/LacB (Additional file 3: Table S2). Under the control of the same promoter, $\mathrm{P}_{L R A 3}$, the trend in $L a c B$ expression was consistent with that of $L R A 3$ (Fig. 4). The production of recombinant protein in $P$. pastoris GS115m/LacB improved with the increase of $\mathrm{P}_{L R A 3}$ transcription activity.

\section{Declined cell viability in P. pastoris GS115m/LacB grown on rhamnose}

Generally, rhamnose metabolism was down-regulated due to the decreased expression of the rate-limiting enzyme LRA4. Low rhamnose metabolism was accompanied by low energy supply and reduced sources of carbon-based biomass components. This resulted in decreased growth rate and declined cell biomass in $P$. pastoris GS115m/LacB grown in YPR medium, which was confirmed by the results of our previous study (Yan et al. 2018). Additionally, it was reported that cell viability was also affected by carbon starvation such as glucose shortage (Oda et al. 2015).

To confirm whether cell viability of $P$. pastoris GS115m/LacB altered, cell growth assay was performed. Differences in the number and size of cell colonies indicated the various profiles of cell viability and generation time of the tested strains, respectively. Small colonies as well as decreased number of cell colonies were observed in $P$. pastoris $\mathrm{GS} 115 \mathrm{~m} / \mathrm{LacB}$ compared with $P$. pastoris GS115/LacB when rhamnose as the carbon source (YPR

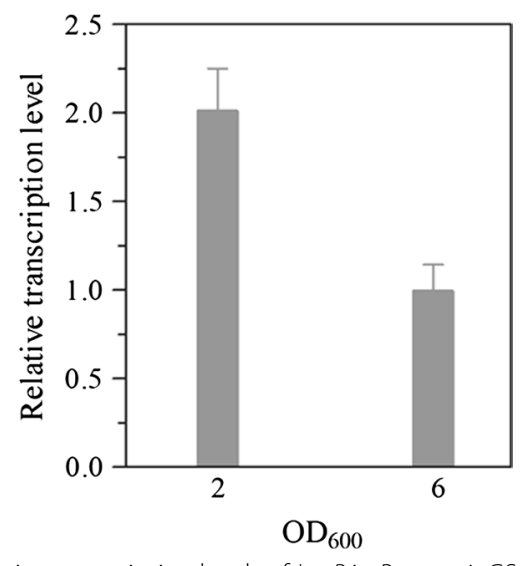

Fig. 4 Relative transcription levels of $L a c B$ in P. pastoris GS115m/LacB grown in YPR medium. The P. pastoris GS115m/LacB strain was grown in liquid YPR medium to an $\mathrm{OD}_{600}$ of $\sim 2$ and $\sim 6$, respectively. The relative expression value of $L a C B$ in P. pastoris GS115/LacB was used as the control and designated as 1 
and MR) (Fig. 5), which indicated prolonged propagation and declined viability in P. pastoris GS115m/LacB cells. This led to a lower biomass of P. pastoris GS115m/LacB grown in YPR and MR, which was described in another study (Yan et al. 2018).

\section{Increased autophagy level in P. pastoris GS115m/LaCB during growth on rhamnose}

Autophagy is a principal catabolic pathway for degrading cellular components including organelles and dysfunctional proteins. Some nonessential cellular components can be degraded via autophagy to synthesize critical components (Devenish and Prescott 2015; Olsvik et al. 2019). Autophagy occurs at low levels under normal conditions (Huang et al. 2015) and increases under adverse conditions such as nutrient deficiency, hypoxia, and oxidative stress (Onodera and Ohsumi 2005; Scherz-Shouval and Elazar 2011; Shpilka et al. 2015; Weidberg et al. 2011). Similarly, autophagy might be induced by the insufficient carbon metabolism due to the decreased rhamnose utilization.

To investigate that autophagy could be triggered by the mild carbon starvation, the autophagy in $P$. pastoris GS115/LacB and P. pastoris GS115m/LacB cells was monitored using autophagosomes staining. Intensive autophagy signals were detected in $P$. pastoris $\mathrm{GS} 115 \mathrm{~m} / \mathrm{LacB}$ cells compared with $P$. pastoris GS115/LacB cells. Obviously, the carbon starvation arose from the decrease of rhamnose metabolism indeed caused autophagy in P. pastoris GS115m/LacB (Fig. 6). We assumed that $P$. pastoris $\mathrm{GS} 115 \mathrm{~m} / \mathrm{LacB}$ cells recycled non-essential components to synthesize essential components for cell survival and reduce cell apoptosis via autophagy under carbon starvation.

\section{Undetectable effect on cell apoptosis due to decreased LRA4 expression}

Autophagy is interconnected with apoptosis because both of them might be triggered by same signals. Autophagy happened in P. pastoris GS115m/LacB, and apoptosis was thereby concerned. In order to understand whether apoptosis underwent in $P$. pastoris GS1 $15 \mathrm{~m} / \mathrm{LacB}$, apoptosis profiles in cells of $P$. pastoris $\mathrm{GS} 115 \mathrm{~m} / \mathrm{LacB}$ and $P$. pastoris GS115/LacB was analyzed by flow cytometry using the Annexin V-FITC/PI apoptosis detection kit. Relatively low apoptosis level occurred in both kinds of cells, and no differences were observed in them (Fig. 7). It indicated that apoptosis did not obviously occur in cells of $P$. pastoris GS115m/LacB and $P$. pastoris GS115/LacB although the intensive autophagy occurred in $P$. pastoris GS115m/LacB. The results showed that the decreased rhamnose metabolism only led to a mild carbon source starvation, which was different from the carbon source starvation due to depletion of carbon source, and brought to a slight alteration of physiological state of $P$. pastoris $\mathrm{GS} 115 \mathrm{~m} / \mathrm{LacB}$ instead of death such as apoptosis.

\section{Low level of reactive oxygen species (ROS) in P. pastoris GS115m/LaCB}

ROS, which at a high level can induce apoptosis (Sullivan and Chandel 2014), was elevated in production when cells survived nutrient starvation such as inadequate supply of glucose (Wang et al. 2018b). Apoptosis did not occur in $P$. pastoris $\mathrm{GS} 115 \mathrm{~m} / \mathrm{LacB}$, indicating a low level of ROS in P. pastoris GS115m/LacB cells. The low levels of ROS in both kinds of cells were further confirmed by flow cytometry after DHR123 staining (Fig. 8).

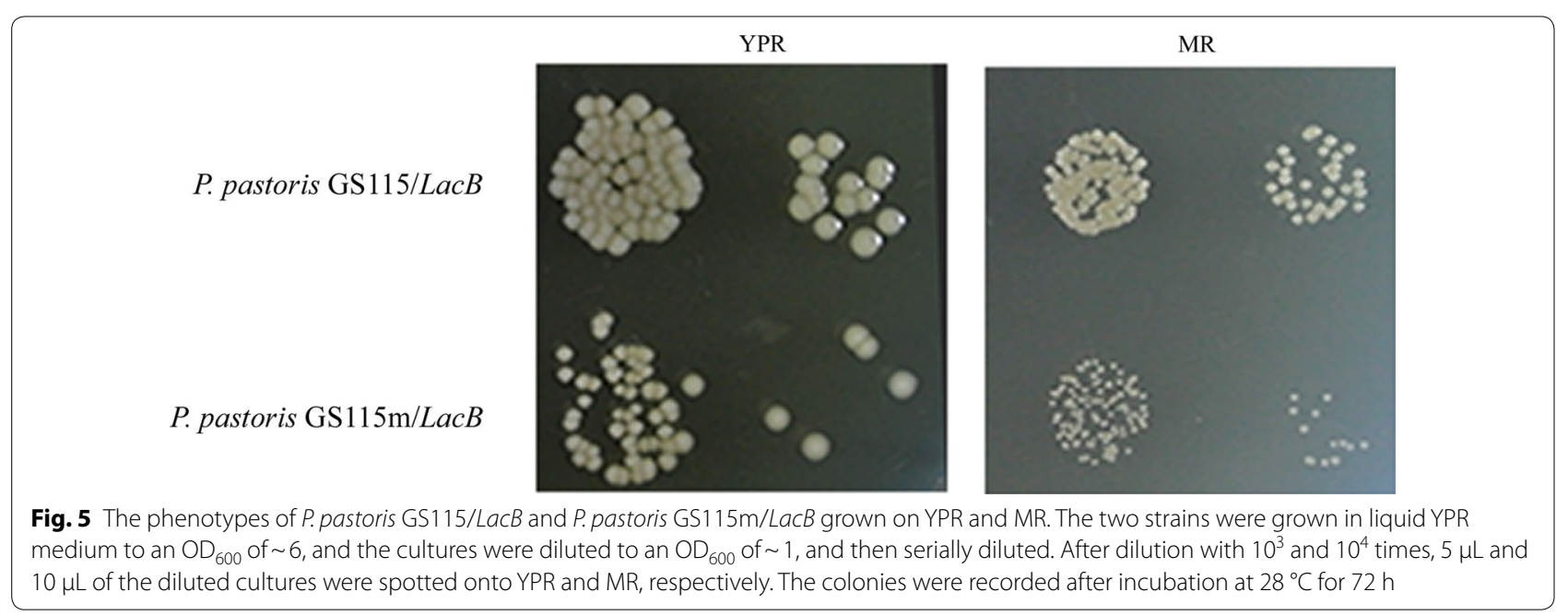




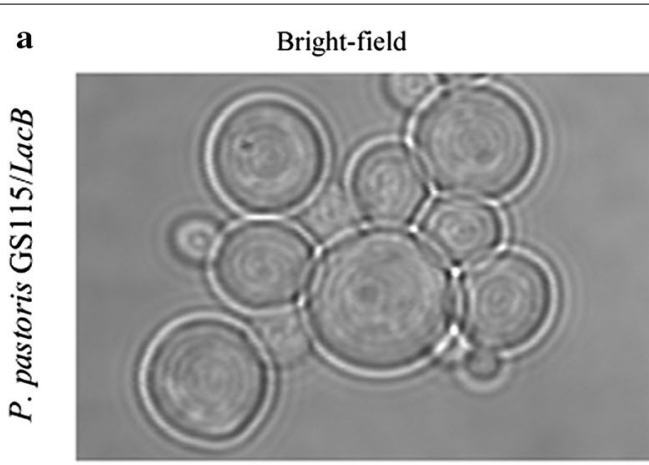

c

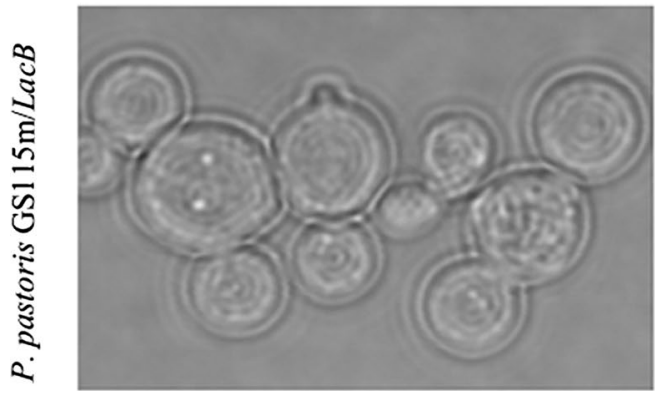

b

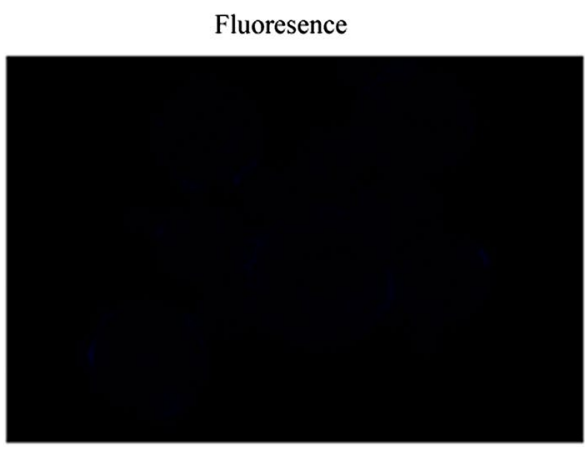

d

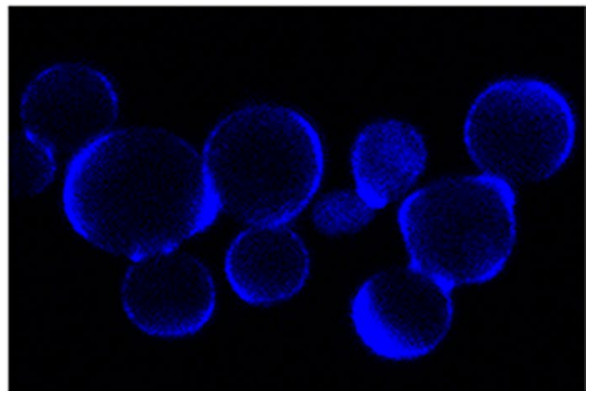

Fig. 6 Autophagy profiles in P. pastoris GS115/LacB and P. pastoris GS115m/LacB cells. The two strains were grown in YPR to an $\mathrm{OD}_{600}$ of $\sim 2$, and then visualized by laser scanning confocal microscope with a $405 \mathrm{~nm}$ excitation wavelength and an emission wavelength shifting from blue $(\sim 450 \mathrm{~nm})$ after staining with Autophagy Assay Kit
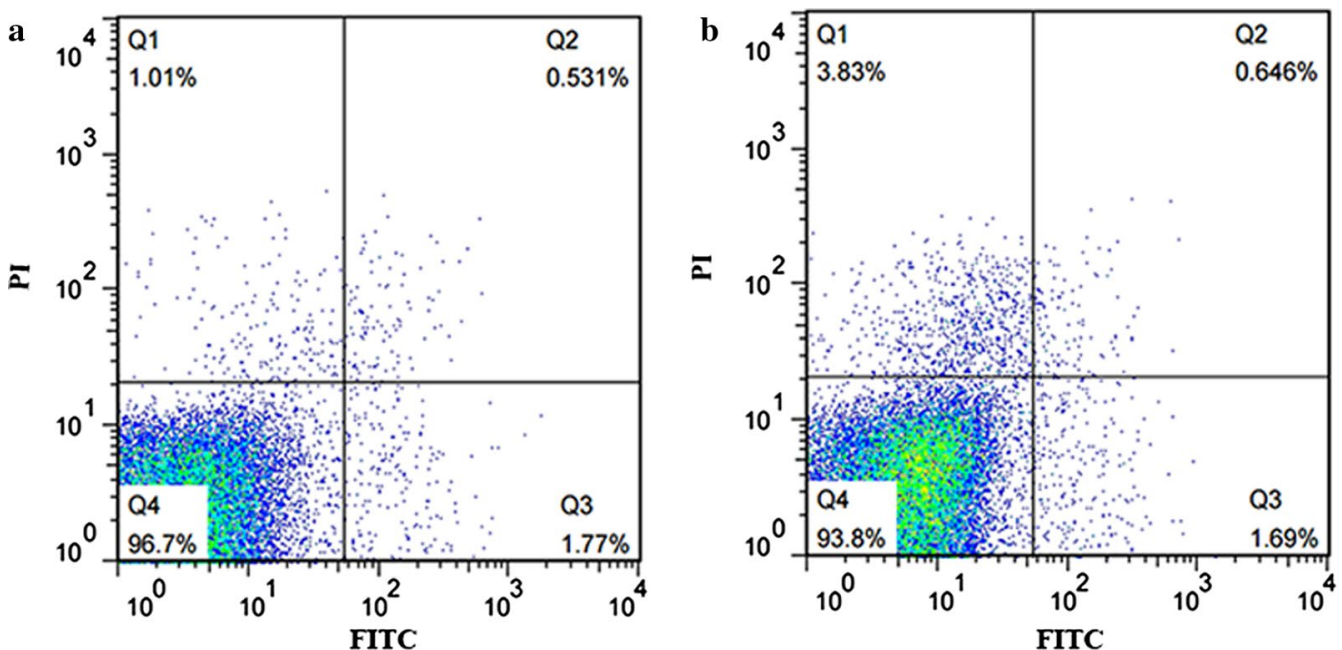

Fig. 7 Cell apoptosis profiles of P. pastoris GS115/LaCB (a) and P. pastoris GS115m/LacB (b), respectively. Fluorescence intensities in 20,000 cells stained with the Annexin V-FITC/PI apoptosis detection kit were monitored by flow cytometry

\section{Discussion}

Rational metabolic engineering has been adopted to enhance the production of target products (Maervoet et al. 2016; Song and Lee 2015), such as deleting the bypass pathway or/and strenghening the precursor synthetic pathway (Fan et al. 2016). In the $\mathrm{P}_{L R A 3}$ system, recombinant proteins were the primary products, and production of recombinant proteins was directly related to the transcriptional activity of $\mathrm{P}_{L R A 3} . \mathrm{P}_{L R A 3}$ activity was positively correlated to two factors, rhamnose concentration and rhamnose induction duration. We considered that the increase in the two factors could be 

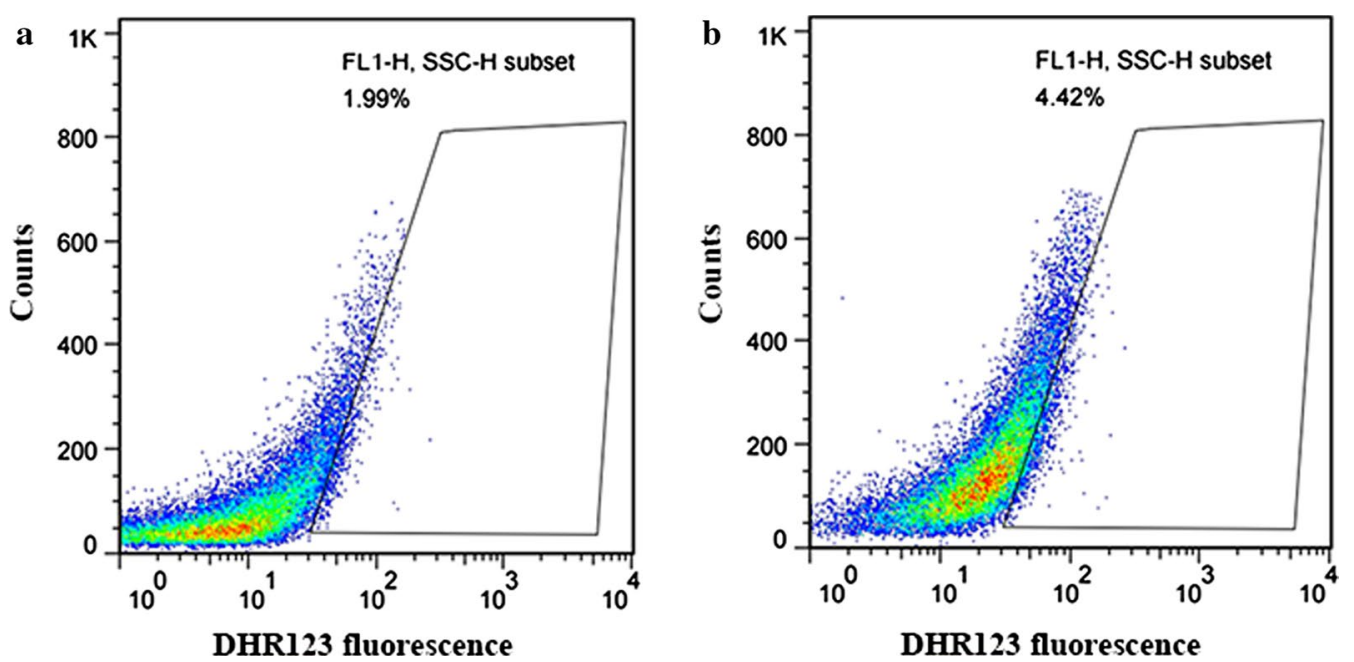

Fig. 8 The intracellular ROS in P. pastoris GS115/LacB (a) and P. pastoris GS115m/LacB (b) using DHR-123. Fluorescence intensities in 20,000 cells were monitored by flow cytometer

realized via decreased metabolism, so the engineering strain was developed by down-regulating the expression of one of key rhamnose utilization related genes, LRA4. As expected, the engineering strains presented the expected profile, increasing the production of target proteins. However, the actual mechanism on the improved production was not elucidated in detail.

In this study, $P_{L R A 3}$ activity in $P$. pastoris $\mathrm{GS} 115 \mathrm{~m} / \mathrm{LacB}$ as well as $P$. pastoris $\mathrm{GS} 115 / \mathrm{LacB}$ was disclosed by determining the transcriptional level of $L R A 3$ in the transcriptome. $\mathrm{P}_{L R A 3}$ was found to be one of the strongest promoters in the presence of rhamnose, and this indicated that recombinant proteins would be highly expressed under the control of $\mathrm{P}_{L R A 3}$. Simultaneously, it was noted that $\mathrm{P}_{L R A 3}$ activity was lower than that of $\mathrm{P}_{G A P}$ in the parental strain while higher in the engineering strain, suggesting an improved transcriptional level of $\mathrm{P}_{L R A 3}$ in the engineering strain. Transcriptional level of recombinant protein gene, $L a c B$, under the control of $\mathrm{P}_{L R A 3}$ was thereby enhanced in the engineering strain, leading to an improved production of LacB. Totally, the elevated $\mathrm{P}_{L R A 3}$ activity directly contributed to the improved production of recombinant proteins.

Rhamnose utilization efficiency in the engineering strain was confirmed to decrease in our previous study. The decreased rhamnose metabolism caused carbon starvation to some extent, and the host would alter some physiological profiles to adapt this kind of stress although this stress was different from the carbon starvation stress arose from glucose depletion. To present, numerous studies have been carried out to investigate carbon starvation due to carbon depletion (Adachi et al. 2017; Marshall and Vierstra 2018; Schwarz et al. 2017), and few reporters have focused specifically on low metabolic flux.

Down-regulated expression of $L R A 4$ exposed P. pastoris $\mathrm{GS} 115 \mathrm{~m} / \mathrm{LacB}$ to a slight carbon starvation stress. As a rescue strategy, P. pastoris $\mathrm{GS} 115 \mathrm{~m} / \mathrm{LacB}$ induced the transcription of rhamnose-utilization genes to increase rhamnose metabolism to attenuate the starvation. In a addition, serious carbon starvation usually brought to some changes of physiological profiles, such as ROS, autophagy, and even apoptosis. Autophagy can block apoptosis to maintain cell survival or induce apoptosis to result in cell death, relying on the nutrient situation. The mild starvation due to insufficient utilization of rhamnose seemed no serious damages to the $P$. pastoris $\mathrm{GS} 115 \mathrm{~m} / \mathrm{LacB}$ cells because only obvious autophagy instead of apoptosis occurred. P. pastoris $\mathrm{GS} 115 \mathrm{~m} / \mathrm{LacB}$ reused some unessential components via autophagy to keep cell viability for survival.

Comprehensively, these findings provided insight into the adaptation mechanisms of microbes under insufficient carbon utilization and some strategies for engineering strain.

\section{Supplementary information}

Supplementary information accompanies this paper at https://doi. org/10.1186/s13568-020-00971-2.

Additional file 1: Table S1. Primers used for real-time PCR.

Additional file 2: Figure S1. Comparison of gene expression between two biological replicates in RNA-seq analysis.

Additional file 3: Table S2. The 25 most highly expressed genes in $P$. pastoris GS115/LaCB and P. pastoris GS115m/LaCB $\left(\mathrm{OD}_{600} \sim 2\right)$. 
Additional file 4: Table S3. The 25 most highly transcribed genes in $P$. pastoris GS115/LacB and P. pastoris GS115m/LacB $\left(\mathrm{OD}_{600} \sim 6\right)$.

\section{Acknowledgements}

Not applicable.

\section{Authors' contributions}

$B L$ and WZ designed the study; JJ, SW, HT, XX and YZ performed experiments; $J$ J, SW, BL and WZ drafted the manuscript. All authors reviewed the results and participated in the writing of the manuscript. All authors read and approved the final manuscript.

\section{Funding}

This study was funded by the National Natural Science Foundation of China (Grant number: 31671802), National Transgenic Major Program (2019ZX08010004) and Central Public-interest Scientific Institution Basal Research Fund (No. Y2019XK19).

\section{Availability of data and materials}

The RNA-seq data were deposited in the CNGBdb (https://db.cngb.org) with accession number CNP0000622 and CNP0000710. The authors declare that all data supporting the findings of this study are available from the corresponding authors upon request.

\section{Ethics approval and consent to participate}

Not applicable.

\section{Consent for publication}

Not applicable.

\section{Competing interests}

The authors declare that have no competing interests.

Received: 6 November 2019 Accepted: 11 February 2020

Published online: 25 February 2020

\section{References}

Adachi A, Koizumi M, Ohsumi Y (2017) Autophagy induction under carbon starvation conditions is negatively regulated by carbon catabolite repression. J Biol Chem 292(48):19905-19918

Ahn J, Hong J, Park M, Lee H, Lee E, Kim C, Lee J, Choi ES, Jung JK, Lee H (2009) Phosphate-responsive promoter of a Pichia pastoris sodium phosphate symporter. Appl Environ Microb 75(11):3528-3534

Anders S, Pyl PT, Huber W (2015) HTSeq-a Python framework to work with high-throughput sequencing data. Bioinformatics 31(2):166-169

Bolger AM, Lohse M, Usadel B (2014) Trimmomatic: a flexible trimmer for illumina sequence data. Bioinformatics 30(15):2114-2120

Devenish RJ, Prescott M (2015) Autophagy: starvation relieves transcriptional repression of ATG genes. Curr Biol 25(6):R238-R240

Fan Y, Hu F, Wei L, Bai L, Hua Q (2016) Effects of modulation of pentosephosphate pathway on biosynthesis of ansamitocins in Actinosynnema pretiosum. J Biotechnol 230:3-10

Huang HH, Kawamata T, Horie T, Tsugawa H, Nakayama Y, Ohsumi Y, Fukusaki E (2015) Bulk RNA degradation by nitrogen starvation-induced autophagy in yeast. EMBO J 34(2):154-168

Jiao J, Wang S, Liang ML, Zhang YH, Xu XX, Zhang W, Liu B (2019) Basal transcription profiles of the rhamnose-inducible promoter $P_{L R A 3}$ and the development of efficient $P_{L R A 3}$-based systems for markerless gene deletion and a mutant library in Pichia pastoris. Curr Genet 65(3):785-798
Liu B, Zhang YW, Zhang X, Yan CL, Zhang YH, Xu XX, Zhang W (2016) Discovery of a rhamnose utilization pathway and rhamnose-inducible promoters in Pichia pastoris. Sci Rep. 6:27352

Maervoet VET, De Maeseneire SL, Avci FG, Beauprez J, Soetaert WK, De Mey M (2016) High yield 1,3-propanediol production by rational engineering of the 3-hydroxypropionaldehyde bottleneck in Citrobacter werkmanii. Microb Cell Fact 15:23

Marshall RS, Vierstra RD (2018) Proteasome storage granules protect proteasomes from autophagic degradation upon carbon starvation. Elife 7:e34532

Oda A, Takemata N, Hirata Y, Miyoshi T, Suzuki Y, Sugano S, Ohta K (2015) Dynamic transition of transcription and chromatin landscape during fission yeast adaptation to glucose starvation. Genes Cells 20(5):392-407

Olsvik HL, Svenning S, Abudu YP, Brech A, Stenmark H, Johansen T, Mejlvang J (2019) Endosomal microautophagy is an integrated part of the autophagic response to amino acid starvation. Autophagy 15(1):182-183

Onodera J, Ohsumi Y (2005) Autophagy is required for maintenance of amino acid levels and protein synthesis under nitrogen starvation. J Biol Chem 280(36):31582-31586

Scherz-Shouval R, Elazar Z (2011) Regulation of autophagy by ROS: physiology and pathology. Trends Biochem Sci 36(1):30-38

Schwarz V, Andosch A, Geretschlager A, Affenzeller M, Lutz-Meindl U (2017) Carbon starvation induces lipid degradation via autophagy in the model alga Micrasterias. J Plant Physiol 208:115-127

Shpilka T, Welter E, Borovsky N, Amar N, Shimron F, Peleg Y, Elazar Z (2015) Fatty acid synthase is preferentially degraded by autophagy upon nitrogen starvation in yeast. Proc Natl Acad Sci USA 112(5):1434-1439

Song CW, Lee SY (2015) Combining rational metabolic engineering and flux optimization strategies for efficient production of fumaric acid. Appl Microbiol Biotechnol 99(20):8455-8464

Stadlmayr G, Mecklenbrauker A, Rothmuller M, Maurer M, Sauer M, Mattanovich D, Gasser B (2010) Identification and characterisation of novel Pichia pastoris promoters for heterologous protein production. J Biotechnol 150(4):519-529

Sullivan LB, Chandel NS (2014) Mitochondrial reactive oxygen species and cancer. Cancer Metab 2:17

Trapnell C, Pachter L, Salzberg SL (2009) TopHat: discovering splice junctions with RNA-Seq. Bioinformatics 25(9):1105-1111

Trapnell C, Williams BA, Pertea G, Mortazavi A, Kwan G, van Baren MJ, Salzberg SL, Wold BJ, Pachter L (2010) Transcript assembly and quantification by RNA-Seq reveals unannotated transcripts and isoform switching during cell differentiation. Nat Biotechnol 28(5):511-515

Vogl T, Glieder A (2013) Regulation of Pichia pastoris promoters and its consequences for protein production. New Biotechnol 30(4):385-404

Wang GY, Li DL, Miao ZG, Zhang SS, Liang WX, Liu L (2018a) Comparative transcriptome analysis reveals multiple functions for Mhy $1 \mathrm{p}$ in lipid biosynthesis in the oleaginous yeast Yarrowia lipolytica. Biochim Biophy Acta Mol Cell Biol Lipids 1863(1):81-90

Wang L, Minchin RF, Butcher NJ (2018b) Arylamine N-acetyltransferase 1 protects against reactive oxygen species during glucose starvation: role in the regulation of p53 stability. PLoS ONE 13(3):e0193560

Weidberg H, Shvets E, Elazar Z (2011) Biogenesis and cargo selectivity of autophagosomes. Annu Rev Biochem 80:125-156

Yan CL, Xu XX, Zhang X, Zhang YW, Zhang YH, Zhang ZF, Zhang W, Liu B (2018) Decreased rhamnose metabolic flux improved production of target proteins and cell flocculation in Pichia pastoris. Front Microbiol 9:1771

\section{Publisher's Note}

Springer Nature remains neutral with regard to jurisdictional claims in published maps and institutional affiliations. 